\title{
Gauge Theory Model of the Neutrino and New Physics Beyond the Standard Model
}

\author{
Yue-Liang $\mathrm{Wu} *$ \\ KITPC/ITP-CAS \\ Kavli Institute for Theoretical Physics China, \\ Institute of Theoretical Physics \\ Chinese Academy of sciences, \\ Beijing 100190, China
}

(Dated:)

\begin{abstract}
Majorana features of neutrinos and $\mathrm{SO}(3)$ gauge symmetry of three families enable us to construct a gauge model of neutrino for understanding naturally the observed smallness of neutrino masses and the nearly tri-bimaximal neutrino mixing when combining together with the mechanism of approximate global $\mathrm{U}(1)$ family symmetry. The vacuum structure of $\mathrm{SO}(3)$ symmetry breaking is found to play an important role. The mixing angle $\theta_{13}$ and $\mathrm{CP}$-violating phases governed by the vacuum of spontaneous symmetry breaking are in general non-zero and testable experimentally at the allowed sensitivity. The model predicts the existence of vector-like $\mathrm{SO}(3)$ triplet charged leptons and vector-like $\mathrm{SO}(3)$ triplet Majorana neutrinos as well as $\mathrm{SO}(3)$ tri-triplet Higgs bosons, some of them can be light and explored at the colliders LHC and ILC.
\end{abstract}

PACS numbers: $14.60 . \mathrm{Pg}, 12.60 .-\mathrm{i}, 11.30 . \mathrm{Hv}, 14.60 . \mathrm{St}$

*Electronic address: ylwu@itp.ac.cn 
The evidence of massive neutrinos strongly indicates new physics beyond the standard model[1]. The dominance of dark matter in our universe challenges the standard models of both particle physics and cosmology. The current neutrino experimental data[2-10] can well be described by neutrino oscillations via three neutrino mixings 11 13]. The global fit from various experimental data lead to the following constraints on the three mixing angles:

$$
30^{\circ}<\theta_{12}<38^{\circ}, \quad 36^{\circ}<\theta_{23}<54^{\circ}, \quad \theta_{13}<10^{\circ}
$$

and the mass squared differences:

$$
\begin{aligned}
& 7.2 \times 10^{-5} \mathrm{eV}^{2}<\Delta m_{21}^{2}=m_{\nu_{\mu}}^{2}-m_{\nu_{e}}^{2}<8.9 \times 10^{-5} \mathrm{eV}^{2}, \\
& 2.1 \times 10^{-3} \mathrm{eV}^{2}<\Delta m_{32}^{2}=m_{\nu_{\tau}}^{2}-m_{\nu_{\mu}}^{2}<3.1 \times 10^{-3} \mathrm{eV}^{2}
\end{aligned}
$$

at the $99 \%$ confidence level[13].

The most unclear parameter is the mixing angle $\theta_{13}$ which is expected to be measured in near future. Phenomenologically, such mixing angles are consistent with the so-called tri-bimaximal mixing with $\theta_{12}=\sin ^{-1}(1 / \sqrt{3})=35^{\circ}, \theta_{23}=\sin ^{-1}(1 / \sqrt{2})=45^{\circ}$ and $\theta_{13}=0$, which was first proposed by Harrison, Perkins and Scott [14] and investigated further by many groups[15 18]. Great theoretical efforts have been made to obtain such a mixing matrix via imposing various symmetries [19 35]. However, for $\theta_{13}=0$, it is hard to be directly tested experimentally. Recently, it was shown that the tri-bimaximal neutrino mixing matrix may be yielded as the lowest order approximation from diagonalizing a special symmetric mass matrix for the Dirac-type neutrinos with a new symmetry[36] and the Majorana-type neutrinos with the $Z_{3}$ group[37], where the mixing angle $\theta_{13}$ is in general non-zero and can be at the experimentally allowed sensitivity. Nevertheless, in comparison with the quark sector, one still faces a puzzle that why neutrino masses are so tiny, but their mixings are so large. It is known that the only peculiar property for neutrinos is that they could be Majorana fermions. It is then natural to conjecture that a solution to the puzzle is most likely attributed to the Majorana features. Thus revealing the origin of large mixing angles and small masses of neutrinos is important not only for understanding neutrino physics, but also for exploring new physics beyond the standard model.

The greatest success of the standard model (SM) is the gauge symmetry structure $S U(3)_{c} \times S U_{L}(2) \times U_{Y}(1)$ which has been tested by more and more precise experiments. As a simple extension of the standard model with three families and Majorana neutrinos, we are 
going to consider in this note a non-abelian gauge family symmetry $\mathrm{SO}(3)$ instead of discrete symmetries discussed widely in literature. It is noted that only $\mathrm{SO}(3)$ rather than $\mathrm{SU}(3)$ is allowed due to the Majorana feature of neutrinos. In fact, it was shown that $\mathrm{SO}(3)$ family symmetry can easily explain the maximal mixing between muon-neutrino and tau-neutrino, i.e., $\theta_{23}=45^{\circ}$ and nearly degenerate neutrino masses[38 44], it can also lead to a nearly bimaximal neutrino mixing (based on the early data) relying on the $\mathrm{SO}(3)$ triplet scalar fields and the symmetry breaking scenarios of SO(3) symmetry[45-47]. As the current experimental data favor a nearly tri-bimaximal mixing, we shall construct in this note an alternative model with gauge symmetry $S O(3) \times S U(3)_{c} \times S U_{L}(2) \times U_{Y}(1)$. The new model contains a minimal set of new particles which include vector-like $\mathrm{SO}(3)$ triplet Majorana fermion and vector-like $\mathrm{SO}(3)$ triplet charged lepton, two $S U(2)_{L}$ Higgs doublets, two $\mathrm{SO}(3)$ tri-triplet Higgs bosons and one singlet Higgs boson. By considering an appropriate symmetry breaking scenario of $\mathrm{SO}(3)$ family symmetry, the nearly tri-bimaximal neutrino mixing matrix is naturally obtained. It is different from our previous consideration for obtaining the nearly bimaximal neutrino mixing, where we introduced only the $\mathrm{SO}(3)$ triplet Higgs bosons. In this note, we shall show that it is more natural and simple to introduce $\mathrm{SO}(3)$ tri-triplet Higgs bosons instead of $\mathrm{SO}(3)$ triplet Higgs bosons to obtain the nearly tri-bimaximal neutrino mixing. In particular, the type II like seesaw mechanism is easily realized in such a model, and the smallness of neutrino masses and nearly tri-bimaximal neutrino mixing can well be understood simultaneously via the symmetry breaking scenario of $\mathrm{SO}(3)$ tri-triplet Higgs bosons and the mechanism of approximate global U(1) family symmetry [48 [50] in the Yukawa sector. Some new particles can be light and probed in future experiments.

For our purpose in this note, let us focus on the following $S O(3) \times S U(2)_{L} \times U(1)_{Y}$ invariant Lagrangian for Yukawa interactions of leptons with Majorana neutrinos

$$
\begin{aligned}
\mathcal{L}_{Y} & =y_{\nu} \bar{l} \tilde{H} \nu_{R}+y_{N} \bar{l} H_{N} N+\frac{1}{2} \xi_{N} \bar{N} \Phi_{\nu} N+\frac{1}{2} M_{R} \bar{\nu}_{R} \nu_{R}^{c} \\
& +y_{e} \bar{l} H E+\xi_{e} \phi_{s} \bar{E} e_{R}+\frac{1}{2} \xi_{E} \bar{E} \Phi_{e} E+H . c .
\end{aligned}
$$

with $y_{\nu}, y_{e}, y_{N}, \xi_{e}, \xi_{N}$ and $\xi_{E}$ being the real Yukawa coupling constants. $M_{R}$ is the mass of right-handed Majorana neutrinos. All the fermions $\nu_{L i}, \nu_{R i}, e_{L i}, e_{R i}, E_{i}$ and $N_{i}(i=1,2,3)$ belong to $\mathrm{SO}(3)$ triplets in family space. Where $\bar{l}_{i}=\left(\bar{\nu}_{L i}, \bar{e}_{L i}\right)$ denote $S U_{L}(2)$ doublet leptons, $H$ and $H_{N}$ are $S U_{L}(2)$ doublet Higgs bosons with $\tilde{H}=\tau_{2} H^{*} . \quad \nu_{R i}$ are the right-handed neutrinos with $\nu_{R i}^{c}=c \bar{\nu}_{R i}^{T}$ the charge conjugated ones. $E_{i}$ are $S U_{L}(2)$ singlet vector-like 
charged leptons and the $N_{i}$ are $S U_{L}(2)$ singlet vector-like Majorana neutrinos with $N_{i}^{c}=N_{i}$. $\phi_{s}$ is a singlet Higgs boson. The scalar fields $\Phi_{\nu}$ and $\Phi_{e}$ are $\mathrm{SO}(3)$ tri-triplets Higgs bosons. The hermiticity condition of Lagrangian and the Majorana condition of vector-like neutrinos imply that

$$
\Phi_{\nu}=\Phi_{\nu}^{*}, \quad \Phi_{\nu}=\Phi_{\nu}^{T}, \quad \Phi_{e}=\Phi_{e}^{\dagger}
$$

Namely $\Phi_{\nu}$ is a real symmetric tri-triplet Higgs boson and contains six independent scalar fields, $\Phi_{e}$ is an Hermitian tri-triplet Higgs boson and contains nine independent scalar fields. With the above fields, the Lagrangian in eq.(1) is the most general one ensured by the following discrete symmetry $\left(Z_{2}\right.$ and $\left.Z_{4}\right)$

$$
N \rightarrow i \gamma_{5} N, \quad \Phi_{\nu} \rightarrow-\Phi_{\nu}, \quad H_{N} \rightarrow-i H_{N}, \quad \phi_{s} \rightarrow-\phi_{s}, \quad e_{R} \rightarrow-e_{R}
$$

We now discuss the interesting features of $\mathrm{SO}(3)$ gauge symmetry. In terms of $\mathrm{SO}(3)$ representation, one can reexpress the real symmetric tri-triplet Higgs boson into the following general form

$$
\Phi_{\nu} \equiv O_{\nu} \phi_{\nu} O_{\nu}^{T}, \quad O_{\nu}(x)=e^{i \lambda^{i} \Theta_{i}^{\nu}(x)}, \quad \phi_{\nu}(x)=\left(\begin{array}{ccc}
\phi_{1}^{\nu} & \phi_{2}^{\nu} & \phi_{3}^{\nu} \\
\phi_{2}^{\nu} & \phi_{3}^{\nu} & \phi_{1}^{\nu} \\
\phi_{3}^{\nu} & \phi_{1}^{\nu} & \phi_{2}^{\nu}
\end{array}\right)
$$

with $\lambda^{i}(i=1,2,3)$ being the generators of $\mathrm{SO}(3)$. Where $\Theta_{i}^{\nu}(x)(i=1,2,3)$ may be regarded as three rotational scalar fields of $\mathrm{SO}(3)$, and $\phi_{i}^{\nu}(x)(i=1,2,3)$ are the remaining three independent scalar fields.

The non-trivial structure of $\phi_{\nu}(x)$ is a unique property of the cyclic Abelian finite group $Z_{3}$ for a real symmetric matrix with three independent elements. A detailed discussion on the properties of $Z_{3}$ group can be found in ref.[37]. The explicit three dimensional unitary representation of $Z_{3}=\left\{t_{i}\right\}(i=1,2,3)$ is given by

$$
t_{1}=\left(\begin{array}{lll}
1 & 0 & 0 \\
0 & 1 & 0 \\
0 & 0 & 1
\end{array}\right), t_{2}=\left(\begin{array}{lll}
0 & 1 & 0 \\
0 & 0 & 1 \\
1 & 0 & 0
\end{array}\right), t_{3}=\left(\begin{array}{lll}
0 & 0 & 1 \\
1 & 0 & 0 \\
0 & 1 & 0
\end{array}\right) .
$$

which is the only nontrivial invariant subgroup of the non-Abelian symmetric group $S_{3}=$ 
$\left\{t_{i}, T_{i}\right\}(i=1,2,3)$ with

$$
T_{1}=\left(\begin{array}{lll}
1 & 0 & 0 \\
0 & 0 & 1 \\
0 & 1 & 0
\end{array}\right), T_{2}=\left(\begin{array}{lll}
0 & 1 & 0 \\
1 & 0 & 0 \\
0 & 0 & 1
\end{array}\right), T_{3}=\left(\begin{array}{lll}
0 & 0 & 1 \\
0 & 1 & 0 \\
1 & 0 & 0
\end{array}\right) .
$$

It is easy to check that the non-trivial structure of $\phi_{\nu}(x)$ belongs to the coset space of the symmetric group $S_{3}$ with $Z_{3}$ as the subgroup, i.e., $\phi_{\nu}(x) \in S_{3} / Z_{3}$. To be more explicit, the symmetric scalar field $\phi_{\nu}(x)$ with three independent components can be expressed in terms of the group representation $\left\{T_{i} \mid i=1,2,3\right\} \in S_{3} / Z_{3}$ as the follows

$$
\phi_{\nu}(x)=\phi_{1}^{\nu}(x) T_{1}+\phi_{2}^{\nu}(x) T_{2}+\phi_{3}^{\nu}(x) T_{3}
$$

which is in a cyclic permuted form $\left[\phi_{\nu}(x)\right]_{i j}=\phi_{i+j-1}^{\nu}(x)$ with $(i+j-1)$ mod. 3 and invariant under the $Z_{3}$ operation

$$
t \phi_{\nu} t=\phi_{\nu}
$$

$\mathrm{SO}(3)$ gauge invariance allows us to fix the gauge by making $\mathrm{SO}(3)$ gauge transformation $g(x)$ to satisfy the condition $g(x) \equiv O_{\nu}(x) \in S O(3)$. With such a gauge fixing, the Yukawa interactions can be rewritten as follows

$$
\begin{aligned}
\mathcal{L}_{Y} & =y_{\nu} \bar{l} \tilde{H} \nu_{R}+y_{N} \bar{l} H_{N} N+\frac{1}{2} \xi_{N} \bar{N} \phi_{\nu} N+\frac{1}{2} M_{R} \bar{\nu}_{R} \nu_{R}^{c} \\
& +y_{e} \bar{l} H E+\xi_{e} \phi_{s} \bar{E} e_{R}+\frac{1}{2} \xi_{E} \bar{E} \hat{\Phi}_{e} E+H . c .
\end{aligned}
$$

where $\hat{\Phi}_{e}=O_{\nu}^{T} \Phi_{e} O_{\nu}$ remains Hermitian and contains nine independent scalar fields. Note that the resulting Lagrangian with such a gauge fixing is invariant under $Z_{3}$ transformation.

The Hermitian $\mathrm{SO}(3)$ tri-triplet scalar field $\hat{\Phi}_{e}(x)$ can generally be reexpressed in terms of $\mathrm{SO}(3)$ representation as the following form

$$
\hat{\Phi}_{e} \equiv U_{e} \phi_{e} U_{e}^{\dagger}, \quad U_{e}(x) \equiv P_{e} O_{e}, \quad O_{e}(x)=e^{i \lambda^{i} \chi_{i}^{e}(x)}
$$

and

$$
P_{e}(x)=\left(\begin{array}{ccc}
e^{i \eta_{1}^{e}(x)} & 0 & 0 \\
0 & e^{i \eta_{2}^{e}(x)} & 0 \\
0 & 0 & e^{i \eta_{3}^{e}(x)}
\end{array}\right), \quad \phi_{e}(x)=\left(\begin{array}{ccc}
\phi_{1}^{e}(x) & 0 & 0 \\
0 & \phi_{2}^{e}(x) & 0 \\
0 & 0 & \phi_{3}^{e}(x)
\end{array}\right)
$$


where $\chi_{i}^{e}(x)(i=1,2,3)$ are regarded as three rotational scalar fields of $\mathrm{SO}(3), \eta_{i}^{e}(x)$ $(i=1,2,3)$ denote three phase scalar fields and $\phi_{i}^{e}(x)(i=1,2,3)$ are the remaining three independent scalar fields.

When all the scalar fields evaluate their vacuum expectation values, both $\mathrm{SO}(3)$ and $S U_{L}(2)$ gauge symmetries and the discrete symmetries are broken down spontaneously. Let us take the previously given gauge fixing condition and consider the following general vacuum structure of scalar fields

$$
\begin{aligned}
& <H(x)>=v, \quad<H_{N}(x)>=v_{N} \\
& <\phi_{s}(x)>=v_{s}, \quad<\phi_{i}^{\nu}(x)>=v_{i}^{\nu}, \\
& <\phi_{i}^{e}(x)>=v_{i}^{e}, \quad<\chi_{i}^{e}(x)>=\theta_{i}^{e}, \quad<\eta_{i}^{e}(x)>=\delta_{i}^{e}
\end{aligned}
$$

namely

$$
<P_{e}>\equiv P_{\delta}^{e}=\operatorname{diag} \cdot\left(e^{i \delta_{1}^{e}}, e^{i \delta_{2}^{e}}, e^{i \delta_{3}^{e}}\right), \quad<O_{e}>=e^{i \lambda^{i} \theta_{i}^{e}}
$$

Where $\delta_{i}^{e}(\mathrm{i}=1,2,3)$ are CP phases arising from spontaneous symmetry breaking and $\theta_{i}^{e}$ are three rotational angles of $\mathrm{SO}(3)$.

With such a vacuum structure after spontaneous symmetry breaking, the mass matrix of neutrinos and charged leptons are given by the following generalized see-saw mechanism

$$
\begin{aligned}
& M_{\nu}=m_{\nu}^{D} M_{R}^{-1} m_{\nu}^{D}+m_{N}^{D} M_{N}^{-1} m_{N}^{D}, \\
& M_{e}=V_{e} m_{E}^{D} M_{E}^{-1} m_{E}^{D} V_{e}^{\dagger}
\end{aligned}
$$

with

$$
\begin{aligned}
& m_{\nu}^{D}=y_{\nu} v, \quad m_{N}^{D}=y_{N} v_{N}, \quad m_{E}^{D}=\sqrt{y_{e} \xi_{e} v v_{s}} \\
& V_{e}=<U_{e}>=P_{\delta}^{e} e^{i \lambda^{i} \theta_{i}^{e}}
\end{aligned}
$$

and

$$
\begin{gathered}
M_{N}=\xi_{N}\left(\begin{array}{ccc}
v_{1}^{\nu} & v_{2}^{\nu} & v_{3}^{\nu} \\
v_{2}^{\nu} & v_{3}^{\nu} & v_{1}^{\nu} \\
v_{3}^{\nu} & v_{1}^{\nu} & v_{2}^{\nu}
\end{array}\right), \quad M_{E}=\xi_{E}\left(\begin{array}{ccc}
v_{1}^{e} & 0 & 0 \\
0 & v_{2}^{e} & 0 \\
0 & 0 & v_{3}^{e}
\end{array}\right) \\
V_{e} \equiv P_{\delta}^{e}\left(\begin{array}{ccc}
c_{12}^{e} c_{13}^{e} & s_{12}^{e} c_{13}^{e} & s_{13}^{e} \\
-s_{12}^{e} c_{23}^{e}-c_{12}^{e} s_{23}^{e} s_{13}^{e} & c_{12}^{e} c_{23}^{e}-s_{12}^{e} s_{23}^{e} s_{13}^{e} & s_{23}^{e} c_{13}^{e} \\
s_{12}^{e} s_{23}^{e}-c_{12}^{e} c_{23}^{e} s_{13}^{e} & -c_{12}^{e} s_{23}^{e}-s_{12}^{e} c_{23}^{e} s_{13}^{e} & c_{23}^{e} c_{13}^{e}
\end{array}\right)
\end{gathered}
$$


Where we have used the notations $c_{i j}^{e} \equiv \cos \theta_{i j}^{e}$ and $s_{i j}^{e} \equiv \sin \theta_{i j}^{e}$. Note that $\theta_{i j}^{e}$ are given as functions of $\theta_{i}^{e}(i=1,2,3)$.

It is of interest to notice that when taking the Majorana neutrino masses $M_{R}$ and $M_{N}$ to be infinity large, the interactions with Majorana neutrinos decouple from the theory. This can be seen from the following effective interactions mediated via the Majorana neutrinos

$$
\frac{y_{\nu}^{2}}{M_{R}} \bar{l} \tilde{H} \tilde{H}^{T} l^{c}, \quad \frac{y_{N}^{2}}{M_{N}} \bar{l} H_{N} H_{N}^{T} l^{c} \rightarrow 0, \quad \text { for } \quad M_{R}, \quad M_{N} \rightarrow \infty
$$

which implies that the resulting Yukawa interactions in this limit generate additional global U(1) family symmetries for the charged lepton sector. Namely, once the Majorana neutrinos become very heavy, the Yukawa interactions possess approximate global U(1) family symmetries.

When applying the mechanism of approximate global $U(1)$ family symmetries [48 50] to the Yukawa interactions after $\mathrm{SO}(3)$ symmetry is broken down spontaneously, we arrive at the following conditions

$$
M_{R} \gg m_{\nu}^{D}, \quad M_{N} \gg m_{N}^{D}, \quad \theta_{i}^{e} \ll 1,
$$

Namely

$$
M_{\nu} \ll 1, \quad \theta_{i j}^{e} \ll 1
$$

which provides a possible explanation why the observed left-handed neutrinos are so light and meanwhile the charged lepton mixing angles must be small. With the above conditions, the neutrino mass matrix (eq.(13)) is given by a type II like see-saw mechanism and the charged lepton mass matrix (eq.(14)) is also presented by a generalized see-saw mechanism. By diagonalizing the mass matrices, we have

$$
V_{\nu}^{T} M_{\nu} V_{\nu}=\operatorname{diag} \cdot\left(m_{\nu_{e}}, m_{\nu_{\mu}}, m_{\nu_{\tau}}\right), \quad V_{e}^{\dagger} M_{e} V_{e}=\operatorname{diag} .\left(m_{e}, m_{\mu}, m_{\tau}\right)
$$

where

$$
V_{\nu}=\left(\begin{array}{ccc}
\frac{2}{\sqrt{6}} c_{\nu} & \frac{1}{\sqrt{3}} & \frac{2}{\sqrt{6}} s_{\nu} \\
-\frac{1}{\sqrt{6}} c_{\nu}-\frac{1}{\sqrt{2}} s_{\nu} & \frac{1}{\sqrt{3}} & \frac{1}{\sqrt{2}} c_{\nu}-\frac{1}{\sqrt{6}} s_{\nu} \\
-\frac{1}{\sqrt{6}} c_{\nu}+\frac{1}{\sqrt{2}} s_{\nu} & \frac{1}{\sqrt{3}} & -\frac{1}{\sqrt{2}} c_{\nu}-\frac{1}{\sqrt{6}} s_{\nu}
\end{array}\right) \equiv V_{0} V_{1}
$$


with

$$
V_{0}=\left(\begin{array}{ccc}
\frac{2}{\sqrt{6}} & \frac{1}{\sqrt{3}} & 0 \\
-\frac{1}{\sqrt{6}} & \frac{1}{\sqrt{3}} & \frac{1}{\sqrt{2}} \\
-\frac{1}{\sqrt{6}} & \frac{1}{\sqrt{3}} & -\frac{1}{\sqrt{2}}
\end{array}\right), \quad V_{1}=\left(\begin{array}{ccc}
c_{\nu} & 0 & s_{\nu} \\
0 & 1 & 0 \\
-s_{\nu} & 0 & c_{\nu}
\end{array}\right)
$$

Here $V_{0}$ is the so-called tri-bimaximal mixing matrix[14]. For short, we have introduced the notations $c_{\nu} \equiv \cos \theta_{\nu}$ and $s_{\nu} \equiv \sin \theta_{\nu}$ with

$$
\tan 2 \theta_{\nu}=\frac{\sqrt{3}\left(v_{21}^{\nu}-v_{31}^{\nu}\right)}{v_{21}^{\nu}+v_{31}^{\nu}}, \quad v_{21}^{\nu} \equiv v_{2}^{\nu}-v_{1}^{\nu}, \quad v_{31}^{\nu} \equiv v_{3}^{\nu}-v_{1}^{\nu}
$$

As a consequence, the leptonic CKM-type mixing matrix in the mass eigenstates of leptons is given by

$$
\begin{aligned}
V=V_{e}^{\dagger} V_{\nu} & \left(\begin{array}{ccc}
c_{12}^{e} c_{13}^{e} & -s_{12}^{e} c_{23}^{e}-c_{12}^{e} s_{23}^{e} s_{13}^{e} & s_{12}^{e} s_{23}^{e}-c_{12}^{e} c_{23}^{e} s_{13}^{e} \\
s_{12}^{e} c_{13}^{e} & c_{12}^{e} c_{23}^{e}-s_{12}^{e} s_{23}^{e} s_{13}^{e} & -c_{12}^{e} s_{23}^{e}-s_{12}^{e} c_{23}^{e} s_{13}^{e} \\
s_{13}^{e} & s_{23}^{e} c_{13}^{e} & c_{23}^{e} c_{13}^{e}
\end{array}\right) \\
& \times\left(\begin{array}{ccc}
e^{-i \delta_{1}^{e}} & 0 & 0 \\
0 & e^{-i \delta_{2}^{e}} & 0 \\
0 & 0 & e^{-i \delta_{3}^{e}}
\end{array}\right)\left(\begin{array}{ccc}
\frac{2}{\sqrt{6}} & \frac{1}{\sqrt{3}} & 0 \\
-\frac{1}{\sqrt{6}} & \frac{1}{\sqrt{3}} & \frac{1}{\sqrt{2}} \\
-\frac{1}{\sqrt{6}} & \frac{1}{\sqrt{3}} & -\frac{1}{\sqrt{2}}
\end{array}\right)\left(\begin{array}{ccc}
c_{\nu} & 0 & s_{\nu} \\
0 & 1 & 0 \\
-s_{\nu} & 0 & c_{\nu}
\end{array}\right) \\
& \equiv P_{\beta}^{e}\left(\begin{array}{cccc}
s_{12} c_{13} & s_{13} e^{-i \delta} \\
-s_{12} c_{23}-c_{12} s_{23} s_{13} e^{i \delta} & c_{12} c_{23}-s_{12} s_{23} s_{13} e^{i \delta} & s_{23} c_{13} \\
s_{12} s_{23}-c_{12} c_{23} s_{13} e^{i \delta} & -c_{12} s_{23}-s_{12} c_{23} s_{13} e^{i \delta} & c_{23} c_{13}
\end{array}\right)\left(\begin{array}{ccc}
e^{i \alpha_{1}} & 0 & 0 \\
0 & e^{i \alpha_{2}} & 0 \\
0 & 0 & 1
\end{array}\right)
\end{aligned}
$$

with $P_{\beta}^{e}=\operatorname{diag} .\left(e^{i \beta_{1}^{e}}, e^{i \beta_{2}^{e}}, e^{i \beta_{3}^{e}}\right)$, and $c_{i j} \equiv \cos \theta_{i j}$ and $s_{i j} \equiv \sin \theta_{i j}$. Where $\beta_{i}^{e}(\mathrm{i}=1,2,3)$ are three phases introduced to parameterize the leptonic mixing matrix into a familiar form which has been used widely for quark mixing matrix. The phases $\alpha_{1}$ and $\alpha_{2}$ are known as Majorana phases. One can relate three mixing angles $\theta_{i j}$ and $\mathrm{CP}$ phases $\beta_{i}^{e}, \delta, \alpha_{1}$ and $\alpha_{2}$ with mixing angles $\theta_{i j}^{e}, \theta_{\nu}$, phases $\delta_{i}^{e}$. Three phases $\beta_{i}^{e}$ can in principle be absorbed by the phase redefinitions of charged leptons. Nevertheless, unlike in the standard model, the phases $\beta_{i}^{e}$ cannot be rotated away in the model due to $\mathrm{SO}(3)$ gauge interactions among three families, their physical effects will occur in processes involving $\mathrm{SO}(3)$ gauge interactions.

It is seen that the smallness of $\theta_{i j}^{e}(i<j)$ is attributed to the mechanism of approximate global U(1) family symmetries in the Yukawa sector. Thus in a good approximation up to the first order of $s_{i j}^{e}$, the leptonic mixing matrix may be expressed as the following simplified 
form

$$
V \simeq\left(\begin{array}{ccc}
1 & -s_{12}^{e} & -s_{13}^{e} \\
s_{12}^{e} & 1 & -s_{23}^{e} \\
s_{13}^{e} & s_{23}^{e} & 1
\end{array}\right) P_{\delta}^{e \dagger}\left(\begin{array}{ccc}
\frac{2}{\sqrt{6}} & \frac{1}{\sqrt{3}} & 0 \\
-\frac{1}{\sqrt{6}} & \frac{1}{\sqrt{3}} & \frac{1}{\sqrt{2}} \\
-\frac{1}{\sqrt{6}} & \frac{1}{\sqrt{3}} & -\frac{1}{\sqrt{2}}
\end{array}\right)\left(\begin{array}{ccc}
c_{\nu} & 0 & s_{\nu} \\
0 & 1 & 0 \\
-s_{\nu} & 0 & c_{\nu}
\end{array}\right)
$$

The three vector-like heavy Majorana neutrino masses are obtained via diagonalizing the mass matrix $M_{N}$, i.e., $V_{\nu}^{\dagger} M_{N} V_{\nu}=\operatorname{diag} .\left(m_{N_{1}}, m_{N_{2}}, m_{N_{3}}\right)$

$$
m_{N_{1}}=-m_{N} \sqrt{1-\Delta}, \quad m_{N_{2}}=m_{N}, \quad m_{N_{3}}=m_{N} \sqrt{1-\Delta}
$$

with

$$
\begin{aligned}
& m_{N} \equiv \xi_{N}\left(v_{1}^{\nu}+v_{2}^{\nu}+v_{3}^{\nu}\right) \\
& \Delta=3\left(v_{1}^{\nu} v_{2}^{\nu}+v_{2}^{\nu} v_{3}^{\nu}+v_{3}^{\nu} v_{1}^{\nu}\right) /\left(v_{1}^{\nu}+v_{2}^{\nu}+v_{3}^{\nu}\right)^{2},
\end{aligned}
$$

Note that the minus sign in $m_{N_{1}}$ occurs when $\left(v_{2}+v_{3}\right)>2 v_{1}$ and can be absorbed by the redefinition of the Majorana neutrino $N_{1} \rightarrow i \gamma_{5} N_{1}$. If $\left(v_{2}+v_{3}\right)<2 v_{1}$, the mass of $N_{3}$ becomes negative. It is noticed that the Majorana neutrinos $N_{1}$ and $N_{3}$ are in general degenerate in their masses in the physical basis.

The masses of three left-handed light Majorana neutrinos are given in the physics basis as follows

$$
\begin{aligned}
& m_{\nu_{e}}=\bar{m}_{0}-m_{1}(2+\bar{\Delta}) \\
& m_{\nu_{\mu}}=\bar{m}_{0} \\
& m_{\nu_{\tau}}=\bar{m}_{0}+m_{1} \bar{\Delta}
\end{aligned}
$$

with

$$
\begin{array}{lc}
\bar{m}_{0} \equiv m_{0}+m_{1}, & \bar{\Delta}=1 / \sqrt{1-\Delta}-1 \\
m_{0}=\left(m_{\nu}^{D}\right)^{2} / M_{R}, & m_{1}=\left(m_{N}^{D}\right)^{2} / m_{N},
\end{array}
$$

With the above analysis, it enables us from the experimentally measured neutrino mass squire differences to extract two mass parameters $m_{0}$ and $m_{1}$ for a given value of parameter $\Delta$ with $\Delta \neq 1[51]$. To be explicit, in terms of the parameter $\bar{\Delta}$ and the neutrino mass squire differences, we obtain the following relations

$$
m_{1}=\sqrt{\left(m_{\nu_{\tau}}^{2}-m_{\nu_{\mu}}^{2}\right) /\left(\left(2 R_{1}+\bar{\Delta}\right) \bar{\Delta}\right)}, \quad m_{0}=\left(R_{1}-1\right) m_{1}
$$


with

$$
\begin{aligned}
& R_{1}=\left(1+\frac{\bar{\Delta}}{2}+\frac{\bar{\Delta}^{2}}{2(2+\bar{\Delta})} R_{0}\right) /\left(1-\frac{\bar{\Delta}}{2+\bar{\Delta}} R_{0}\right) \\
& R_{0}=\left(m_{\nu_{\mu}}^{2}-m_{\nu_{e}}^{2}\right) /\left(m_{\nu_{\tau}}^{2}-m_{\nu_{\mu}}^{2}\right)
\end{aligned}
$$

For a numerical calculation, we now present the light neutrino masses as functions of the parameter $\Delta$ by taking the central values of the mass squire differences $\Delta m_{21}^{2}=m_{\nu_{\mu}}^{2}-m_{\nu_{e}}^{2}=8 \times 10^{-5} e V^{2}$ and $\Delta m_{32}^{2}=m_{\nu_{\tau}}^{2}-m_{\nu_{\mu}}^{2}=2.5 \times 10^{-3} \mathrm{eV}^{2}$ (see table 1).

Table 1: The masses of neutrino mass eigenstates $\nu_{e}, \nu_{\mu}, \nu_{\tau}$ and mass parameters $m_{0}$ and $m_{1}$ as the functions of the vacuum parameter $\Delta$

\begin{tabular}{|c|c|c|c|c|c|}
\hline$\Delta$ (input) & $m_{0}\left(10^{-2} \mathrm{eV}\right)$ & $m_{1}\left(10^{-2} \mathrm{eV}\right)$ & $m_{\nu_{e}}\left(10^{-2} \mathrm{eV}\right)$ & $m_{\nu_{\mu}}\left(10^{-2} \mathrm{eV}\right)$ & $m_{\nu_{\tau}}\left(10^{-2} \mathrm{eV}\right)$ \\
\hline 0.75 & 1.297 & 2.487 & -3.677 & 3.784 & 6.271 \\
0.73 & 1.271 & 2.637 & -3.908 & 4.003 & 6.346 \\
0.71 & 1.245 & 2.789 & -4.034 & 4.333 & 6.424 \\
0.69 & 1.220 & 2.943 & -4.163 & 4.666 & 6.506 \\
\hline
\end{tabular}

where the minus sign of the Majorana neutrino mass can be absorbed by a redefinition of the neutrino field $\nu_{e} \rightarrow i \nu_{e}$.

From the definition of the parameter $\Delta$ (eq.(31)), it is determined by two ratios $v_{1}^{\nu} / v_{2}^{\nu}$ and $v_{3}^{\nu} / v_{2}^{\nu}$ of three vacuum expectation values $v_{i}^{\nu}(i=1,2,3)$. Also the mixing angle $\theta_{\nu}$ given in eq. $(25)$ is determined by the same two ratios. To relate the mixing angle $\theta_{\nu}$ with the parameter $\Delta$ under the condition $v_{2}^{\nu}+v_{3}^{\nu}>2 v_{1}^{\nu}$, it is reasonable to consider the interesting case with $v_{1}^{\nu} \ll v_{2}^{\nu}, v_{3}^{\nu}$. In this case, the ratio $v_{3}^{\nu} / v_{2}^{\nu}$ becomes dominant and the parameter $\Delta$ gets the maximal value $\Delta \leq 3 / 4$ at $v_{3}^{\nu}=v_{2}^{\nu}$. Explicitly, we have the following approximate relations when $v_{1}^{\nu} \ll v_{2}^{\nu}, v_{3}^{\nu}$

$$
\Delta \simeq \frac{3 r}{(1+r)^{2}}, \quad \tan 2 \theta_{\nu} \simeq \frac{\sqrt{3}(1-r)}{1+r}, \quad r \equiv v_{3}^{\nu} / v_{2}^{\nu}
$$

which shows that in this case both the mixing angle $\theta_{\nu}$ and the ratio $r$ can be determined for the given values of $\Delta$. In the special case that $\Delta=r$, we arrive at the solution

$$
\Delta=r=\sqrt{3}-1, \quad \tan 2 \theta_{\nu}=2-\sqrt{3}, \quad \theta_{\nu}=7.5^{\circ}
$$


Let us now consider the leptonic mixing matrix element $V_{13}$ which has the following general form

$$
V_{13} \simeq \frac{2}{\sqrt{6}} s_{\nu} e^{-i \delta_{1}^{e}}+\frac{1}{\sqrt{2}} c_{\nu}\left(s_{13}^{e} e^{-i \delta_{3}^{e}}-s_{12}^{e} e^{-i \delta_{2}^{e}}\right) \equiv s_{13} e^{-i \delta_{1}^{e}-i \delta_{\nu}}
$$

Here the definition of phase $\delta_{\nu}$ is, by convention, more useful for discussing CP violation in neutrino sector. It is seen that to completely determine the mixing angle $\theta_{13}$ and the CP-violating phase $\delta_{\nu}$, one needs to know the charged lepton mixing angles $s_{12}^{e}$ and $s_{13}^{e}$, the relative $\mathrm{CP}$ phases $\left(\delta_{1}^{e}-\delta_{2}^{e}\right)$ and $\left(\delta_{1}^{e}-\delta_{3}^{e}\right)$. For a numerical estimation, it is useful to investigate the following two interesting cases

$$
\begin{array}{ll}
\text { Case I : } & s_{13}^{e} \simeq 0, s_{12}^{e} \simeq 0 \\
\text { Case II }: & s_{13}^{e} \ll s_{12}^{e} \sim \sqrt{m_{e} / m_{\mu}} \simeq 0.07, \quad \delta_{1}^{e}-\delta_{2}^{e}=\pi / 2
\end{array}
$$

Here the Case I ignores the charged lepton mixing angles for the simplicity of analysis. The Case II is a typical case for the small charged lepton mixing with assuming a maximal CP-violating phase $\left(\delta_{1}^{e}-\delta_{2}^{e}\right)=\pi / 2$. Though we are not able to precisely predict the mixing angle $\theta_{13}$ and the CP-violating phase $\delta_{\nu}$, while from the two typical cases, it allows us to present a reasonable estimation for $\theta_{13}$ and $\delta_{\nu}$ (see table 2 ).

Table 2

\begin{tabular}{|c|c|c|c|c|c|c|}
\hline$\Delta$ (input) & $r=v_{3}^{\nu} / v_{2}^{\nu}$ & $\theta_{\nu}$ & $\theta_{13}($ Case I) & $\delta_{\nu}($ Case I) & $\theta_{13}($ Case II $)$ & $\delta_{\nu}($ Case II $)$ \\
\hline 0.75 & 1.0 & 0 & 0 & 0 & $2.8^{\circ}$ & $90^{\circ}$ \\
0.748 & 0.90 & $2.6^{\circ}$ & $2.1^{\circ}$ & 0 & $3.5^{\circ}$ & $54^{\circ}$ \\
0.745 & 0.85 & $4.0^{\circ}$ & $3.3^{\circ}$ & 0 & $4.4^{\circ}$ & $41^{\circ}$ \\
0.74 & 0.79 & $5.8^{\circ}$ & $4.7^{\circ}$ & 0 & $5.5^{\circ}$ & $31^{\circ}$ \\
0.73 & 0.72 & $7.9^{\circ}$ & $6.4^{\circ}$ & 0 & $7.0^{\circ}$ & $24^{\circ}$ \\
0.71 & 0.63 & $10.7^{\circ}$ & $8.7^{\circ}$ & 0 & $9.2^{\circ}$ & $18^{\circ}$ \\
0.69 & 0.56 & $13.0^{\circ}$ & $10.6^{\circ}$ & 0 & $11.0^{\circ}$ & $15^{\circ}$ \\
\hline
\end{tabular}

which indicates that as long as $v_{3}^{\nu} \neq v_{2}^{\nu}$ (i.e., $r \neq 1$ ) which should be a more general and reasonable case when no symmetry is imposed, the mixing angle $\theta_{13}$ can be large enough to be detected. For the case II, both mixing angle $\theta_{13}$ and CP-violating phase $\delta_{\nu}$ are in general testable by the future neutrino experiments. 
From the above analysis, it is seen that as a reasonable consideration that $v_{1}^{\nu} \ll v_{2}^{\nu}$, $v_{3}^{\nu}$ with $v_{3}^{\nu} \neq v_{2}^{\nu}$, one only needs to take the parameter $\Delta$ to be slightly away from its maximal value $\Delta=0.75$. Numerically, taking $\Delta \simeq 0.72 \pm 0.028$, we then arrive at the most optimistic predictions for the mixing angle $\theta_{13}$ and $\mathrm{CP}$-violating phase $\delta_{\nu}$

$$
\theta_{13} \simeq 7^{\circ} \pm 4^{\circ}, \quad \delta_{\nu} \simeq 35^{\circ} \pm 20^{\circ}, \quad r=v_{3}^{\nu} / v_{2}^{\nu} \simeq 0.73 \pm 0.17
$$

which can be tested in the future experiments. It is seen that the resulting value for the ratio $r=v_{3}^{\nu} / v_{2}^{\nu}$ is also reasonable.

We now turn to discuss the possible mass scales of new physics beyond the standard model. When taking the Dirac neutrino masses $m_{\nu}^{D}$ to be at the order of $(0.1 \sim 1.0) \mathrm{MeV}$ (i.e., at the same order of electron mass), the mass scale $M_{R}$ of right-handed heavy Majorana neutrino $\nu_{R}$ is found, from the values of $m_{0}$ in table 1 , to be

$$
M_{R} \simeq O(1 \sim 100) \mathrm{TeV}
$$

Similarly, if taking the Dirac type neutrino mass $m_{N}^{D}$ to be at the same order $m_{N}^{D} \simeq(0.1 \sim$ 1.0) $\mathrm{MeV}$, the vector-like Majorana neutrino masses are resulted from the values of $m_{1}$ in table 1 to be

$$
\begin{aligned}
& m_{N_{2}} \equiv m_{N} \simeq 0.5 M_{R} \simeq O(500) \mathrm{GeV} \sim O(50) \mathrm{TeV} \\
& m_{N_{1}}=m_{N_{3}}=(0.50 \sim 0.56) m_{N_{2}} \simeq O(250) \mathrm{GeV} \sim O(25) \mathrm{TeV}
\end{aligned}
$$

which shows that the vector-like Majorana neutrino masses can be at the electroweak scale in this case. In general, their masses can be expected to be much smaller if the discrete symmetry imposed in eq.(3) is only softly broken.

The masses for the vector-like charged leptons and their relations to the SM charged leptons are given as follows

$$
\begin{aligned}
& m_{E_{i}}=\xi_{E} v_{i}^{e}, \quad(i=1,2,3) \\
& m_{E_{1}}=\left(m_{E}^{D}\right)^{2} / m_{e}, \quad m_{E_{2}}=\left(m_{E}^{D}\right)^{2} / m_{\mu}, \quad m_{E_{3}}=\left(m_{E}^{D}\right)^{2} / m_{\tau}
\end{aligned}
$$

which shows that the vector-like charged lepton masses have opposite hierarchy to the SM charged lepton masses. It is seen that the heavy triplet charged lepton masses depend on the mass parameter $m_{E}^{D}$. For a numerical estimation, taking $m_{E}^{D} \simeq(15 \sim 25) \mathrm{GeV}$, we obtain the lightest vector-like charged lepton mass $m_{E_{3}}$ to be

$$
m_{E_{3}} \simeq(127 \sim 352) \mathrm{GeV}
$$


which can be explored at LHC and ILC. The other two vector-like charged leptons become very heavy with masses above $\mathrm{TeV}$ scale.

In conclusion, we have shown that the nearly tri-bimaximal neutrino mixings and the smallness of neutrino masses can simultaneously be understood in a flavor dynamical model with $\mathrm{SO}(3)$ gauge family symmetry. It has been seen that the vacuum structure of $\mathrm{SO}(3)$ symmetry breaking for the $\mathrm{SO}(3)$ tri-triplet Higgs bosons and the mechanism of approximate global U(1) family symmetry play an important role. The type II like seesaw mechanism is realized in such a model. The mixing angle $\theta_{13}$ is in general nonzero and its values can range from the experimentally allowed sensitivity to the current experimental bound. CP violation in the lepton sector is caused by a spontaneous symmetry breaking and can be significantly large. Both the mixing angle $\theta_{13}$ and $\mathrm{CP}$-violating phase $\delta_{\nu}$ are expected to be testable by the future more precise neutrino experiments. Some of the predicted vector-like charged leptons and vector-like Majorana neutrinos as well as new Higgs bosons in this model can be explored at the future experiments as their masses can approach to the current experimental bounds. In particular, the mechanism discussed in this note can be extended to the quark sector for understanding the smallness of quark mixing angles, which will be investigated elsewhere.

\section{Acknowledgments}

This work was supported in part by the National Science Foundation of China (NSFC) under the grant 10475105, 10491306, and the key Project of Chinese Academy of Sciences (CAS).

[1] For a recent review see: R. N. Mohapatra and A. Y. Smirnov, Ann. Rev. Nucl. Part. Sci. 56 $569(2006)$.

[2] B. Aharmim et al. (SNO Collaboration), Phys.Rev. C72 (2005) 055502.

[3] K. Eguci et al. (KamLAND Collaboration), Phys. Rev. Lett. 94, 081801(2005).

[4] E.Aliu et al.(K2K Collaboration), Phys. Rev. Lett. 94, 081802(2005)

[5] Y. Ashie et al. ( SK Collaboration), Phys.Rev. D71 (2005).

[6] M. Altmann et al. (GNO Collaboration), Phys. Lett. B616, 174(2005). 
[7] M. Ambrosio et al. (MARCO Collaboration), Eur. Phys. J. C36, 323(2004).

[8] M. Sanchez et al.( Soudan 2 Collaboration), Phys. Rev. D68, 113004(2003).

[9] M. Apollonio et al. (CHOOZ Collaboration), Eur. Phys. J. C27, 331(2003).

[10] W.-M. Yao, et al., Particle Data Group, Journal of Phys. G33, 1 (2006).

[11] M. Maltoni, T. Schwetz, M. A. Tortotla and J. W. F. Valle, Phys. Rev. D68, 113010 (2003).

[12] G.L. Fogli, E. Lisi, A. Marrone, A. Palazzo, Prog. Part. Nucl. Phys. 57 (2006) 742-795.

[13] A. Strumia and F. Vissani, arXiv:hep-ph/0606054.

[14] P. F. Harrison, D. H. Perkins and W. G. Scott, Phys. Lett. B 530, 167 (2002).

[15] Z.-Z. Xing, Phys. Lett. B533, 85(2002).

[16] P. F. Harrison and W.G. Scott, Phys. Lett. B535, 163(2002).

[17] P.F. Harrison and W.G. Scott, Phys. Lett. B557, 76(2003).

[18] X. G. He and A. Zee, Phys. Lett. B560, 87(2003).

[19] C.I. Low and R. R. Volkas, Phys. Rev. D68, 033007 (2003).

[20] E. Ma, Phys. Rev. D70, 031901R(2004);

[21] G. Altarelli and F. Feruglio, Nucl. Phys. B720, 64(2005);

[22] E. Ma, Phys. Rev. D72, 037301 (2005).

[23] E. Ma, Mod. Phys. Lett. A 20, 2601 (2005).

[24] A. Zee, Phys. Lett. B630, 58 (2005).

[25] E. Ma, Phys. Rev. D 73, 057304 (2006).

[26] G. Altarelli and F. Feruglio, Nucl. Phys. B741, 215(2006).

[27] W. Grimus and L. Lavoura, JHEP, 0601:018(2006).

[28] J.E. Kim and J.-C. Park, JHEP 0605:017(2006).

[29] N. Singh, M. Rajkhowa and A. Borach, hep-ph/0603189.

[30] R. Mohapatra, S. Naris and Y.-H. Yu, Phys.Lett. B639 318 (2006).

[31] P. Kovtun and A. Zee, Phys.Lett. B640 (2006) 37.

[32] N. Haba, A. Watanabe and K. Yoshioka, Phys.Rev.Lett. 97 (2006) 041601.

[33] X.G. He, Y.Y. Keum and R. Volkas, JHEP, 0604:039(2006).

[34] I. Varizelas, S.-F. King and G.G. Ross, Phys.Lett. B644 (2007) 153.

[35] E.Ma, hep-ph/0701016.

[36] R. Friedberg and T. D. Lee, arXiv:hep-ph/0606071; arXiv:hep-ph/0705.4156.

[37] B.Hu, F. Wu and Y.L. Wu, Phys.Rev. D75 113003 (2007). 
[38] Y.L. Wu, Phys.Rev. D60 (1999) 073010.

[39] Y.L. Wu, Nucl.Phys.Proc.Suppl. 85 (2000) 193.

[40] Y.L. Wu, invited talk at the 30th International Conference on High-Energy Physics (ICHEP 2000), Osaka, Japan.

[41] C. Carone and M. Sher, Phys. Lett. B420, 83 (1998).

[42] E. Ma, hep-ph/9812344.

[43] C. Wetterich, hep-ph/9812426.

[44] R. Barbieri, L.J. Hall, G.L. Kane and G.G. Ross, hep-ph/9901228.

[45] Y.L. Wu, Eur.Phys.J. C10 (1999) 491.

[46] Y.L. Wu, J. Phys. G: Nucl. Part. Phys. 261131 (2000).

[47] Y.L. Wu, Science in China A43 (2000) 988.

[48] L.J. Hall and S. Weinberg, Phys. Rev. D48, 979 (1993).

[49] Y.L. Wu and L. Wolfenstein, Phys. Rev. Lett. 731762 (1994).

[50] Y.L. Wu, Carnegie-Mellon Univ. report, CMU-HEP94-01, hep-ph/9404241, 1994;

Invited talk at 5th Conference on the Intersections of Particle and Nuclear Physics, St. Petersburg, FL, 31 May- 6 Jun 1994, published in Proceedings, pp338, edited by S.J. Seestrom (AIP, New York, 1995), hep-ph/9406306.

[51] Note that when all the vacuum expectation values are equal, i.e., $v_{1}^{\nu}=v_{2}^{\nu}=v_{3}^{\nu}$, the vector-like Majorana neutrino mass matrix becomes democratic and $\Delta=1$. As a consequence, two of the vector-like Majorana neutrinos become massless in the physical basis and the type II like see-saw mechanism cannot be realized to obtain the observed neutrino masses and mixing. 